\title{
The Association Between Short-term Exposure to Ambient Air Pollution and Patient-Level Home Blood Pressure Among Patients With Chronic Cardiovascular Diseases in a Web-Based Synchronous Telehealth Care Program: Retrospective Study
}

Ching-Chang Huang ${ }^{1,2}$, MD; Ying-Hsien Chen ${ }^{1,2}, \mathrm{MD}$; Chi-Sheng Hung ${ }^{1,2}, \mathrm{MD}, \mathrm{PhD}$; Jen-Kuang Lee ${ }^{1,2}, \mathrm{MD}$, PhD; Tse-Pin Hsu ${ }^{1,3}$, MSN; Hui-Wen Wu ${ }^{1,3}$, MSN; Pao-Yu Chuang ${ }^{1,3}$, MSN; Ming-Fong Chen ${ }^{1,2}$, MD, PhD; Yi-Lwun $\mathrm{Ho}^{1,2}, \mathrm{MD}, \mathrm{PhD}$

\footnotetext{
${ }^{1}$ Telehealth Center, National Taiwan University Hospital, Taipei, Taiwan

${ }^{2}$ Department of Internal Medicine, National Taiwan University Hospital, Taipei, Taiwan

${ }^{3}$ Department of Nursing, National Taiwan University Hospital, Taipei, Taiwan
}

Corresponding Author:

Yi-Lwun Ho, MD, PhD

Telehealth Center

National Taiwan University Hospital

7 Chung-Shan South Road

Taipei, 10002

Taiwan

Phone: 886223123456 ext 63737

Fax: 886223216938

Email: ylho@ntu.edu.tw

\section{Abstract}

Background: The association between short-term exposure to ambient air pollution and blood pressure has been inconsistent, as reported in the literature.

Objective: This study aimed to investigate the relationship between short-term ambient air pollution exposure and patient-level home blood pressure (HBP).

Methods: Patients with chronic cardiovascular diseases from a telehealth care program at a university-affiliated hospital were enrolled as the study population. HBP was measured by patients or their caregivers. Hourly meteorological data (including temperature, relative humidity, wind speed, and rainfall) and ambient air pollution monitoring data (including $\mathrm{CO}, \mathrm{NO}_{2}$, particulate matter with a diameter of $<10 \mu \mathrm{m}$, particulate matter with a diameter of $<2.5 \mu \mathrm{m}$, and $\mathrm{SO}_{2}$ ) during the same time period were obtained from the Central Weather Bureau and the Environmental Protection Administration in Taiwan, respectively. A stepwise multivariate repeated generalized estimating equation model was used to assess the significant factors for predicting systolic and diastolic blood pressure (SBP and DBP).

Results: A total of 253 patients and 110,715 HBP measurements were evaluated in this study. On multivariate analysis, demographic, clinical, meteorological factors, and air pollutants significantly affected the HBP (both SBP and DBP). All 5 air pollutants evaluated in this study showed a significant, nonlinear association with both home SBP and DBP. Compared with demographic and clinical factors, environmental factors (meteorological factors and air pollutants) played a minor yet significant role in the regulation of HBP.

Conclusions: Short-term exposure to ambient air pollution significantly affects HBP in patients with chronic cardiovascular disease.

(JMIR Public Health Surveill 2021;7(6):e26605) doi: 10.2196/26605

\section{KEYWORDS}

ambient air pollution; blood pressure; cardiovascular disease; chronic disease; climate; home blood pressure; particulate matter; pollution; remote monitoring; telehealth care; telemonitoring; weather 


\section{Introduction}

Air pollution is a great hazard to public health [1-5]. Previous studies have shown that long-term exposure to ambient air pollution increases respiratory morbidity and cardiopulmonary disease-related mortality [6,7], while short-term exposure increases cardiovascular disease-related mortality and nonfatal events [8]. The most prominent air pollutants that potentially affect human health include nitrogen oxides (NOx, including $\mathrm{NO}_{2}$ and $\mathrm{NO}$ ), $\mathrm{CO}, \mathrm{O}_{3}, \mathrm{SO}_{2}$, particulate matter with a diameter of $<10 \mu \mathrm{m}\left(\mathrm{PM}_{10}\right)$, and particulate matter with a diameter of $<2.5 \mu \mathrm{m}\left(\mathrm{PM}_{2.5}\right)$. Hypertension is among the most prominent risk factors contributing to cardiovascular diseases, and the potential detrimental effect of air pollution on blood pressure (BP) is considered one of the mechanisms underlying the exacerbation in cardiovascular outcomes. However, the association between short-term or long-term exposure to ambient air pollution and BP has been inconsistent in previous studies, and most of these studies were epidemiological [9-12]. In this retrospective study, we aimed to evaluate the effect of short-term exposure to ambient air pollution on patient-level home blood pressure (HBP).

\section{Methods}

\section{Patient Population}

We retrospectively enrolled patients with chronic cardiovascular diseases (CVDs) who participated in telehealth care at National Taiwan University Hospital (NTUH), Taipei, Taiwan, between January 2009 and December 2013 as the study population. We excluded patients who did not reside in Taipei City during the study period. Informed consent was obtained from all participants. The study was approved by the institutional review board of NTUH. Chronic CVDs included coronary artery disease, prior myocardial infarction, heart failure, peripheral artery disease, prior stroke, and hypertension.

\section{Telehealth Care Program}

In this study, we used a fourth-generation telehealth program developed by Anker et al [13]. The program was designed specifically to offer home care for chronic CVDs. We had previously described the details of this program [14-18]. Briefly, single-lead electrocardiography, BP, heart rate, and oxygen saturation were measured at home, daily and on demand, and the data were instantaneously transmitted to the NTUH Telehealth Center. Case managers would call patients or their caregivers daily and on demand to evaluate patient conditions and provide instructions as needed. There was a call center, and full-time case managers and in-charge cardiologists were available 24 hours a day, 7 days a week. Medical decisions were discussed with patients' primary care physicians in the case of any major acute event.

\section{Data Collection}

Demographic, clinical (diagnosis of specific diseases), and medication data were obtained from the electronic database of NTUH. All biometric data were measured at home and automatically transmitted to the NTUH server. Biometric data were meant to be measured at least twice daily, ideally after waking up and before sleeping, but each patient or caregiver could have his/her own habit to select a time point and interval within a day to measure biometric parameters. We used the AViTA BPM65ZB sphygmomanometer (AViTA Corp), which is an electronic digital upper arm BP monitor. Hourly meteorological data (including temperature, relative humidity, and wind speed) of Taipei City during the study period were obtained from the Central Weather Bureau, Taiwan. Hourly ambient air pollution monitoring data (including $\mathrm{CO}, \mathrm{NO}_{2}$, $\mathrm{PM}_{10}, \mathrm{PM}_{2.5}$, and $\mathrm{SO}_{2}$ ) during the same study period were obtained from the Environmental Protection Administration, Taiwan.

\section{Statistical Analysis}

Statistical analysis was performed using the R software (version 3.4.2, The R Foundation for Statistical Computing). In statistical testing, a 2 -sided $P$ value of $\leq .05$, was considered significant. The distribution properties of continuous variables are expressed as mean (SD) and median (IQR) values, and categorical variables are presented as frequencies and percentages. The differences in the distributions of continuous variables between male and female subjects were examined using the Wilcoxon rank-sum test. Differences in the distributions of categorical variables were compared using the Fisher exact test. Multivariate analysis was conducted by fitting multiple linear regression models to estimate the adjusted effects of age, sex, comorbidities (including hypertension, diabetes mellitus, cancers, atrial fibrillation, heart failure, prior myocardial infarction, coronary artery disease, prior stroke, and peripheral artery disease), heart rate, antihypertensive agents, seasons, meteorological factors, air pollutants, and other predictors of home systolic and diastolic blood pressure (SBP and DBP).

Since the use of antihypertensive agents, values of meteorological factors, and concentrations of air pollutants varied over time, we defined and included the following three groups of time-dependent covariates in our linear regression analyses:

1. Antihypertensive agents: among the 6 most common classes of antihypertensive drugs, we considered the classes of antihypertensive medications and the number of classes of antihypertensive medications used on the day of BP measurement.

2. Meteorological factors: these included hourly averaged outdoor temperature, relative humidity, and wind speed within the hour of BP measurement.

3. Air pollutants: we determined hourly inverse-distance weighted mean concentrations of 5 air pollutants $\left(\mathrm{CO}, \mathrm{NO}_{2}\right.$, $\mathrm{PM}_{10}, \mathrm{PM}_{2.5}$, and $\mathrm{SO}_{2}$ ) and the amount of rainfall within the hour of BP measurement, where the distances were calculated from each patient's home location to the 6 air quality monitoring stations in Taipei City, based on the corresponding latitudes and longitudes. Instant and cumulated air pollutant concentrations at hours $0,3,6,12$, and 18 and days $1,2,3,4,5,6$, and 7 were included in the multivariate analysis to evaluate the possible lag effect of each pollutant. 
Simple and multiple generalized additive models (GAMs) were fitted to assess the nonlinear effects of continuous covariates and identify appropriate cut-off points for discretizing continuous covariates, if necessary, during stepwise variable selection. Further details on statistical analysis are provided in Multimedia Appendix 1.

\section{Results}

\section{Patient Population and Demographics}

A total of 253 patients with CVD who participated in the NTUH Telehealth Care Program from January 2009 to December 2013 were enrolled in this study. A total of 110,715 HBP measurements were carried out accordingly for these patients. The details of this patient population, including the per-patient and per-measurement demographics and clinical characteristics, have already been reported previously [18]. The data distributions of the air pollutant concentrations are summarized in Table 1. The mean temperature, humidity, and wind speed had been reported previously [18]. The mean concentration of $\mathrm{NO}_{2}, \mathrm{PM}_{10}, \mathrm{PM}_{2.5}, \mathrm{CO}$, and $\mathrm{SO}_{2}$ were 23.67 (SD 6.80) ppb, $46.83(\mathrm{SD} 21.03) \mu \mathrm{g} / \mathrm{m}^{3}, 27.96$ (SD 10.77) $\mu \mathrm{g} / \mathrm{m}^{3}, 0.74$ (SD 0.25 ) ppm, and 3.05 (SD 1.19) ppb, respectively. The air quality standards recommended by the World Health Organization for these pollutants, during the study period, were based on the 2005 version of the update [19].

Table 1. Data distribution of air pollutants from among 110,715 observations obtained by 253 patients included in this study.

\begin{tabular}{|c|c|c|c|c|}
\hline Air pollutant & Mean (SD) & Minimum & Maximum & Median \\
\hline $\mathrm{NO}_{2}(\mathrm{ppb})$ & $23.67(6.80)$ & 4.43 & 63.01 & 23.19 \\
\hline $\mathrm{PM}_{10}^{\mathrm{a}}\left(\mu \mathrm{g} / \mathrm{m}^{3}\right)$ & $46.83(21.03)$ & 11.64 & 842.87 & 42.60 \\
\hline $\mathrm{PM}_{2.5}{ }^{\mathrm{b}}\left(\mu \mathrm{g} / \mathrm{m}^{3}\right)$ & $27.96(10.77)$ & 8.16 & 140.39 & 26.07 \\
\hline $\mathrm{CO}(\mathrm{ppm})$ & $0.74(0.25)$ & 0.14 & 2.74 & 0.70 \\
\hline $\mathrm{SO}_{2}(\mathrm{ppb})$ & $3.05(1.19)$ & 0.58 & 14.85 & 2.79 \\
\hline
\end{tabular}

${ }^{\mathrm{a}} \mathrm{PM}_{10}$ : particulate matter with a diameter of $<10 \mu \mathrm{m}$.

${ }^{\mathrm{b}} \mathrm{PM}_{2.5}$ : particulate matter with a diameter of $<2.5 \mu \mathrm{m}$.

\section{Multivariate Analysis}

Multivariate analysis was conducted by fitting multiple linear regression models to estimate the adjusted effects of demographic, clinical, and meteorological factors and air pollutants on home SBP and DBP measurements. The use of antihypertensive agents, values of meteorological factors, and concentrations of air pollutants were all defined and computed as time-dependent covariates. Multivariate analysis of the predictors for SBP and DBP on fitting 1 multiple linear regression model with the stepwise variable selection procedure is shown in Tables 2-7. With regard to SBP and DBP, the significant demographic and clinical characteristics are listed in Tables 2 and 5, meteorological factors in Tables 3 and 6, and air pollutants in Tables 4 and 7 , respectively. The hourly averaged temperature had a linear negative effect on both SBP and DBP, while the other meteorological factors had significant nonlinear correlations with home SBP and DBP, as reported previously [18]. The exact statistical data for these meteorological factors were not identical to those reported previously, since this multivariate analysis was performed by using a new model that also included air pollutants. Similarly, $\mathrm{CO}, \mathrm{NO}_{2}, \mathrm{SO}_{2}, \mathrm{PM}_{2.5}$, and $\mathrm{PM}_{10}$ concentrations were significantly correlated with home SBP and DBP, and the effects were considered nonlinear. The cut-off points for discretizing the continuous covariates with nonlinear effects on the mean values of SBP and DBP ( $\mathrm{mmHg}$ ) were determined objectively, using the corresponding GAM plots during stepwise variable selection. The GAM plots representing the relationships between SBP and different environmental factors are shown in Figure 1-5. The bar plots of regression coefficient estimates for home SBP and DBP are shown in Multimedia Appendix 2. The green, blue, and red bars represent the demographic and clinical factors, meteorological parameters, and air pollution parameters, respectively. Of note, although the demographic, clinical, meteorological, and air pollution parameters significantly affected home SBP and DBP, it seemed that the "traditional" factors (demographic and clinical) had a more prominent effect on HBP than the environmental factors.

The multiple linear regression model for SBP $(n=110,715$; $R^{2}=0.1286$ ) indicates that the Pearson correlation coefficient between the observed value of SBP and the model-predicted value of SBP was $0.1286^{1 / 2}=0.3586$, and that for DBP $\left(\mathrm{n}=110,715 ; R^{2}=0.2219\right)$ indicates that the Pearson correlation coefficient between the observed value of DBP and the model-predicted value of DBP was $0.2219^{1 / 2}=0.4711$. 
Table 2. Multivariate analysis of the predictors for systolic blood pressure by fitting 1 multiple linear regression model with stepwise variable selection: demographic and clinical characteristics.

\begin{tabular}{|c|c|c|c|c|}
\hline Covariate & Parameter estimate & SE & $t$ value & $\operatorname{Pr}>|t|$ \\
\hline Intercept & 125.0560 & 0.8216 & 152.2027 & $<.001$ \\
\hline Male & -0.5184 & 0.1133 & -4.5771 & $<.001$ \\
\hline $\mathrm{AF}^{\mathrm{a}}$ & 0.5663 & 0.1370 & 4.1323 & $<.001$ \\
\hline Coronary artery disease without myocardial infarction & 3.0135 & 0.1086 & 27.7503 & $<.001$ \\
\hline Coronary artery disease with myocardial infarction & -0.5264 & 0.1708 & -3.0820 & .002 \\
\hline Cancer & 3.0896 & 0.1402 & 22.0379 & $<.001$ \\
\hline Chronic heart failure & -4.0580 & 0.1228 & -33.0523 & $<.001$ \\
\hline $\mathrm{CVA}^{\mathrm{b}}$ & 1.1025 & 0.1336 & 8.2507 & $<.001$ \\
\hline $\mathrm{PAOD}^{\mathrm{c}}$ & -1.7202 & 0.1855 & -9.2744 & $<.001$ \\
\hline $\mathrm{ARB}^{\mathrm{d}} \times \mathrm{AB}^{\mathrm{e}}$ & 4.1642 & 0.4037 & 10.3161 & $<.001$ \\
\hline $\mathrm{ARB} \times \mathrm{BB}^{\mathrm{f}}$ & 1.8479 & 0.2601 & 7.1044 & $<.001$ \\
\hline $\mathrm{ARB} \times \mathrm{CCB}^{\mathrm{g}}$ & -3.2486 & 0.2218 & -14.6451 & $<.001$ \\
\hline BB & -0.7201 & 0.1743 & -4.1310 & $<.001$ \\
\hline $\mathrm{CCB} \times \mathrm{AB}$ & -8.0115 & 0.3885 & -20.6223 & $<.001$ \\
\hline $\mathrm{CCB} \times \mathrm{ACEI}^{\mathrm{h}}$ & -8.3039 & 1.2350 & -6.7237 & $<.001$ \\
\hline $\mathrm{CCB} \times \mathrm{BB}$ & -3.9889 & 0.2950 & -13.5204 & $<.001$ \\
\hline $\mathrm{CCB} \times$ Diuretics & 1.0011 & 0.2187 & 4.5766 & $<.001$ \\
\hline Diuretics $\times \mathrm{AB}$ & 7.1806 & 0.2805 & 25.6013 & $<.001$ \\
\hline Diuretics $\times$ ACEI & 1.5441 & 0.4555 & 3.3902 & .001 \\
\hline Diuretics $\times \mathrm{BB}$ & 2.0257 & 0.2549 & 7.9458 & $<.001$ \\
\hline
\end{tabular}

${ }^{\mathrm{a}} \mathrm{AF}$ : atrial fibrillation.

${ }^{\mathrm{b}} \mathrm{CVA}$ : cardiovascular accident.

${ }^{\mathrm{c}}$ PAOD: peripheral arterial occlusion disease.

${ }^{\mathrm{d}}$ ARB: angiotensin receptor blocker.

${ }^{\mathrm{e}} \mathrm{AB}$ : alpha blocker.

${ }^{\mathrm{f}} \mathrm{BB}$ : beta blocker.

${ }^{\mathrm{g}} \mathrm{CCB}$ : calcium channel blocker.

${ }^{\mathrm{h}}$ ACEI: angiotensin-converting enzyme inhibitor. 
Table 3. Multivariate analysis of the predictors for systolic blood pressure by fitting 1 multiple linear regression model with stepwise variable selection: meteorological factors.

\begin{tabular}{|c|c|c|c|c|}
\hline Covariate & Parameter estimate & SE & $t$ value & $\operatorname{Pr}>|t|$ \\
\hline Temperature & -0.6352 & 0.0127 & -49.9892 & $<.001$ \\
\hline $\mathrm{DM}^{\mathrm{a}} \times$ temperature & 0.1882 & 0.0045 & 41.4358 & $<.001$ \\
\hline $\mathrm{HTN}^{\mathrm{b}} \times$ temperature & 0.2101 & 0.0047 & 44.5223 & $<.001$ \\
\hline $\mathrm{ARB}^{\mathrm{c}} \times$ temperature & 0.1528 & 0.0057 & 26.6504 & $<.001$ \\
\hline $\mathrm{CCB}^{\mathrm{d}} \times$ temperature & 0.0520 & 0.0078 & 6.6818 & $<.001$ \\
\hline Diuretics $\times$ temperature & -0.0217 & 0.0059 & -3.6496 & $<.001$ \\
\hline $0.557<$ Wind speed 12 hours ago $\leq 3.73$ & 0.3100 & 0.1008 & 3.0752 & .002 \\
\hline $1.976<$ Wind speed on day $0 \leq 4.43$ & 0.5216 & 0.1099 & 4.7471 & $<.001$ \\
\hline $1.983<$ Wind speed 1 day ago $\leq 3.895$ & 0.2267 & 0.1044 & 2.1707 & .03 \\
\hline $1.793<$ Wind speed 2 days ago $\leq 3.634$ & 0.3138 & 0.0990 & 3.1702 & .002 \\
\hline $1.587<$ Wind speed 4 days ago $\leq 3.923$ & 0.2128 & 0.0995 & 2.1380 & .03 \\
\hline $1.855<$ Wind speed 6 days ago $\leq 3.575$ & 0.3155 & 0.0979 & 3.2221 & .001 \\
\hline Relative humidity $\leq 65.774$ or $>84.596$ & 0.5365 & 0.1000 & 5.3641 & $<.001$ \\
\hline Relative humidity 6 hours ago $\leq 72.967$ or $>92.905$ & 0.4753 & 0.1096 & 4.3364 & $<.001$ \\
\hline Relative humidity 12 hours ago $\leq 56.324$ or $>78.989$ & 0.3813 & 0.1178 & 3.2365 & .001 \\
\hline Relative humidity 24 hours ago $>76.11$ & 0.4278 & 0.1209 & 3.5391 & $<.001$ \\
\hline Relative humidity 2 days ago $\leq 67.752$ or $>82.532$ & 0.3446 & 0.1003 & 3.4362 & .001 \\
\hline Relative humidity 4 days ago $\leq 65.366$ or $>82.318$ & 0.4172 & 0.1044 & 3.9960 & $<.001$ \\
\hline Relative humidity 6 days ago $\leq 58.849$ or $>81.108$ & 0.5261 & 0.1331 & 3.9527 & $<.001$ \\
\hline Log rainfall & -0.2074 & 0.0593 & -3.4984 & .001 \\
\hline Log cumulated rainfall in the past 4 days & -0.2981 & 0.0684 & -4.3564 & $<.001$ \\
\hline Log rainfall 6 days ago $<-0.106$ & 0.6989 & 0.1652 & 4.2296 & $<.001$ \\
\hline
\end{tabular}

${ }^{\mathrm{a} D M}$ : diabetes mellitus.

${ }^{\mathrm{b}} \mathrm{HTN}$ : hypertension.

${ }^{\mathrm{c}} \mathrm{ARB}$ : angiotensin receptor blocker.

${ }^{\mathrm{d} C C B}$ : calcium channel blocker. 
Table 4. Multivariate analysis of the predictors for systolic blood pressure by fitting 1 multiple linear regression model with stepwise variable selection: air pollutants.

\begin{tabular}{|c|c|c|c|c|}
\hline Covariate & Parameter estimate & SE & $t$ value & $\operatorname{Pr}>|t|$ \\
\hline Log CO concentration 3 hours ago & -0.6620 & 0.1186 & -5.5800 & $<.001$ \\
\hline Log cumulated CO concentration in the past 5 days & 1.1985 & 0.2965 & 4.0422 & $<.001$ \\
\hline Log $\mathrm{NO}_{2}$ concentration & 0.4520 & 0.1493 & 3.0276 & .003 \\
\hline $2.571<\log \mathrm{NO}_{2}$ concentration 3 hours ago $\leq 3.654$ & 0.3311 & 0.1113 & 2.9752 & .003 \\
\hline Log $\mathrm{NO}_{2}$ concentration 6 hours ago & 0.7517 & 0.1154 & 6.5136 & $<.001$ \\
\hline $2.188<\log \mathrm{NO}_{2}$ concentration 18 hours ago $\leq 3.185$ & 0.4333 & 0.0986 & 4.3971 & $<.001$ \\
\hline $3.062<\log \mathrm{NO}_{2}$ concentration 3 days ago $\leq 3.497$ & 0.4392 & 0.1026 & 4.2810 & $<.001$ \\
\hline $3.063<\log \mathrm{NO}_{2}$ concentration 4 days ago $\leq 3.483$ & 0.3689 & 0.1027 & 3.5929 & $<.001$ \\
\hline $2.567<\log \mathrm{NO}_{2}$ concentration 5 days ago $\leq 3.507$ & 0.4131 & 0.1435 & 2.8781 & .004 \\
\hline $3.059<\log \mathrm{NO}_{2}$ concentration 6 days ago $\leq 3.494$ & 0.4837 & 0.1037 & 4.6650 & $<.001$ \\
\hline $3.043<\log \mathrm{NO}_{2}$ concentration 7 days ago $\leq 3.468$ & 0.3438 & 0.1004 & 3.4256 & .001 \\
\hline $\log \mathrm{PM}_{2.5}{ }^{\mathrm{a}}$ concentration 3 hours ago $<2.284$ & 1.1068 & 0.3245 & 3.4105 & .001 \\
\hline $\log \mathrm{PM}_{2.5}$ concentration 4 days ago $\leq 3.243$ or $>4.378$ & 0.5000 & 0.1031 & 4.8481 & $<.001$ \\
\hline Log $\mathrm{PM}_{2.5}$ concentration 6 days ago $\leq 3.034$ or $>3.826$ & 0.3055 & 0.1076 & 2.8383 & .005 \\
\hline $2.848<\log \mathrm{PM}_{10}{ }^{\mathrm{b}}$ concentration 18 hours ago $\leq 3.828$ & 0.4819 & 0.1032 & 4.6690 & $<.001$ \\
\hline Log $\mathrm{SO}_{2}$ concentration 3 hours ago $<-0.271$ & 3.5314 & 1.0028 & 3.5216 & $<.001$ \\
\hline Log $\mathrm{SO}_{2}$ concentration 24 hours ago $>1.561$ & 0.4552 & 0.1372 & 3.3164 & .001 \\
\hline Log $\mathrm{SO}_{2}$ concentration 5 days ago $>1.316$ & 0.2970 & 0.1187 & 2.5025 & .01 \\
\hline
\end{tabular}

${ }^{\mathrm{a}} \mathrm{PM}_{2.5}$ : particulate matter with a diameter of $<2.5 \mu \mathrm{m}$.

${ }^{\mathrm{b}} \mathrm{PM}_{10}$ : particulate matter with a diameter of $<10 \mu \mathrm{m}$. 
Table 5. Multivariate analysis of the predictors for diastolic blood pressure by fitting 1 multiple linear regression model with stepwise variable selection: demographic and clinical characteristics.

\begin{tabular}{|c|c|c|c|c|}
\hline Covariate & Parameter estimate & SE & $t$ value & $\operatorname{Pr}>|t|$ \\
\hline Intercept & 65.7236 & 0.7686 & 85.5088 & $<.001$ \\
\hline Male & -1.2416 & 0.0793 & -15.6608 & $<.001$ \\
\hline Age $<72.424$ years & 8.5878 & 0.0783 & 109.6523 & $<.001$ \\
\hline $\mathrm{AF}^{\mathrm{a}}$ & -0.8389 & 0.0916 & -9.1609 & $<.001$ \\
\hline Coronary artery disease with myocardial infarction & -0.3166 & 0.1088 & -2.9105 & .004 \\
\hline Cancer & 0.4197 & 0.0942 & 4.4572 & $<.001$ \\
\hline $\mathrm{CHF}^{\mathrm{b}}$ & -0.8912 & 0.0806 & -11.0540 & $<.001$ \\
\hline $\mathrm{CVA}^{\mathrm{c}}$ & 3.2332 & 0.0896 & 36.0651 & $<.001$ \\
\hline PAOD $^{d}$ & 1.8971 & 0.1279 & 14.8373 & $<.001$ \\
\hline $\mathrm{ARB}^{\mathrm{e}} \times \mathrm{AB}^{\mathrm{f}}$ & 6.8305 & 0.2722 & 25.0906 & $<.001$ \\
\hline $\mathrm{ARB} \times \mathrm{BB}^{\mathrm{g}}$ & 5.6929 & 0.1636 & 34.7953 & $<.001$ \\
\hline $\mathrm{ARB} \times \mathrm{CCB}^{\mathrm{h}}$ & -3.3888 & 0.1369 & -24.7458 & $<.001$ \\
\hline $\mathrm{ARB} \times$ diuretics & -3.4067 & 0.1401 & -24.3191 & $<.001$ \\
\hline $\mathrm{CCB} \times \mathrm{ACEI}^{\mathrm{i}}$ & -10.7720 & 0.8297 & -12.9827 & $<.001$ \\
\hline $\mathrm{CCB} \times \mathrm{BB}$ & -0.4592 & 0.1868 & -2.4583 & .01 \\
\hline $\mathrm{CCB} \times$ diuretics & 2.2884 & 0.1298 & 17.6274 & $<.001$ \\
\hline Diuretics $\times \mathrm{AB}$ & -3.4526 & 0.2494 & -13.8457 & $<.001$ \\
\hline Diuretics $\times$ ACEI & 2.5617 & 0.3943 & 6.4973 & $<.001$ \\
\hline Diuretics $\times \mathrm{BB}$ & -1.7401 & 0.1616 & -10.7675 & $<.001$ \\
\hline
\end{tabular}

${ }^{\mathrm{a}} \mathrm{AF}$ : atrial fibrillation.

${ }^{\mathrm{b}} \mathrm{CHF}$ : congestive heart failure.

${ }^{\mathrm{c}} \mathrm{CVA}$ : cerebrovascular accident.

${ }^{\mathrm{d}}$ PAOD: peripheral occlusive arterial disease.

${ }^{\mathrm{e}} \mathrm{ARB}$ : angiotensin receptor blocker.

${ }_{\mathrm{f}}^{\mathrm{AB}}$ : alpha blocker.

${ }^{\mathrm{B}}$ BB: beta blocker.

${ }^{\mathrm{h}} \mathrm{CCB}$ : calcium channel blocker.

${ }^{\mathrm{i}} \mathrm{ACEI}$ : angiotensin converting enzyme inhibitor. 
Table 6. Multivariate analysis of the predictors for diastolic blood pressure by fitting 1 multiple linear regression model with stepwise variable selection: meteorological factors.

\begin{tabular}{|c|c|c|c|c|}
\hline Covariate & Parameter estimate & SE & $t$ value & $\operatorname{Pr}>|t|$ \\
\hline Temperature & -0.1608 & 0.0130 & -12.3600 & $<.001$ \\
\hline Temperature 12 hours ago & -0.1058 & 0.0135 & -7.8265 & $<.001$ \\
\hline Temperature 18 hours ago $\leq 16.992$ or $>28.649$ & 0.5042 & 0.0714 & 7.0590 & $<.001$ \\
\hline Temperature 7 days ago & 0.0753 & 0.0124 & 6.0722 & $<.001$ \\
\hline $\mathrm{DM}^{\mathrm{a}} \times$ temperature & 0.0694 & 0.0030 & 22.9718 & $<.001$ \\
\hline $\mathrm{HTN}^{\mathrm{b}} \times$ temperature & 0.0857 & 0.0032 & 26.9498 & $<.001$ \\
\hline $\mathrm{AB}^{\mathrm{c}} \times$ temperature & -0.1161 & 0.0083 & -13.9588 & $<.001$ \\
\hline ACEI $^{\mathrm{d}} \times$ temperature & -0.0323 & 0.0096 & -3.3598 & .001 \\
\hline $\mathrm{ARB}^{\mathrm{e}} \times$ temperature & 0.0623 & 0.0046 & 13.5546 & $<.001$ \\
\hline Diuretics $\times$ temperature & -0.0177 & 0.0045 & -3.9649 & $<.001$ \\
\hline Wind speed & 0.1122 & 0.0250 & 4.4919 & $<.001$ \\
\hline Wind speed 18 hours ago & 0.0612 & 0.0230 & 2.6618 & .008 \\
\hline Wind speed 3 days ago & 0.1358 & 0.0318 & 4.2695 & $<.001$ \\
\hline Wind speed 5 days ago $>3.734$ & 0.3835 & 0.0892 & 4.3014 & $<.001$ \\
\hline Wind speed 7 days ago $>4.56$ & 0.7900 & 0.1750 & 4.5145 & $<.001$ \\
\hline Relative humidity 6 hours ago $\leq 67.108$ or $>91.436$ & 0.3259 & 0.0719 & 4.5359 & $<.001$ \\
\hline Relative humidity 12 hours ago $\leq 57.1$ or $>78.822$ & 0.2567 & 0.0836 & 3.0700 & .002 \\
\hline Relative humidity 18 hours $\leq 34.851$ or $>77.249$ & 0.2478 & 0.0983 & 2.5216 & .01 \\
\hline Relative humidity 24 hours ago $\leq 47.631$ or $>77.286$ & 0.2813 & 0.0893 & 3.1501 & .002 \\
\hline Relative humidity 2 days ago $\leq 64.191$ or $>81.286$ & 0.2489 & 0.0728 & 3.4188 & .001 \\
\hline Relative humidity 4 days ago $\leq 64.291$ or $>81.392$ & 0.3521 & 0.0729 & 4.8281 & $<.001$ \\
\hline Relative humidity 5 days ago $\leq 54.611$ or $>76.3$ & 0.3089 & 0.0852 & 3.6268 & $<.001$ \\
\hline Relative humidity 6 days ago $\leq 54.383$ or $>79.265$ & 0.5044 & 0.0985 & 5.1202 & $<.001$ \\
\hline Relative humidity 7 days ago $\leq 58.455$ or $>76.46$ & 0.5243 & 0.0856 & 6.1239 & $<.001$ \\
\hline Log rainfall > -2.252 & 0.3004 & 0.0824 & 3.6452 & $<.001$ \\
\hline Log rainfall 3 hours ago $\leq-0.372$ or $>0.817$ & 0.4580 & 0.1538 & 2.9783 & .003 \\
\hline$-2.251<$ Log rainfall 6 hours ago $\leq 0.379$ & 0.3796 & 0.0856 & 4.4340 & $<.001$ \\
\hline$-2.246<$ Log rainfall 12 hours ago $\leq 0.177$ & 0.3282 & 0.0874 & 3.7542 & $<.001$ \\
\hline$-2.228<$ Log rainfall 18 hours ago $\leq 3.202$ & 0.1674 & 0.0846 & 1.9797 & .05 \\
\hline$-2.165<$ Log rainfall 2 days ago $\leq 0.145$ & 0.4425 & 0.0681 & 6.4961 & $<.001$ \\
\hline$-2.168<\log$ rainfall 4 days ago $\leq 0.068$ & 0.3491 & 0.0682 & 5.1162 & $<.001$ \\
\hline$-2.153<$ Log rainfall 5 days ago $\leq 0.026$ & 0.3042 & 0.0691 & 4.4003 & $<.001$ \\
\hline$-2.193<$ Log rainfall 7 days ago $\leq-0.266$ & 0.5065 & 0.0666 & 7.6091 & $<.001$ \\
\hline$-2.044<\log$ cumulated rainfall in the past 6 days $\leq-0.735$ & 0.5517 & 0.0691 & 7.9805 & $<.001$ \\
\hline Log cumulated rainfall in the past 7 days & -0.5756 & 0.0538 & 10.7067 & $<.001$ \\
\hline
\end{tabular}

${ }^{\mathrm{a} D M}$ : diabetes mellitus.

${ }^{\mathrm{b}} \mathrm{HTN}$ : hypertension.

${ }^{\mathrm{c}} \mathrm{AB}$ : alpha blocker.

${ }^{\mathrm{d}}$ ACEI: angiotensin converting enzyme inhibitor. 
Table 7. Multivariate analysis of the predictors for diastolic blood pressure by fitting 1 multiple linear regression model with stepwise variable selection: air pollutants.

\begin{tabular}{|c|c|c|c|c|}
\hline Covariate & Parameter estimate & SE & $t$ value & $\operatorname{Pr}>|t|$ \\
\hline Log CO concentration & 0.3966 & 0.0970 & 4.0907 & $<.001$ \\
\hline Log CO concentration 12 hours ago & -0.9318 & 0.0759 & -12.2815 & $<.001$ \\
\hline$-0.41<\log$ CO concentration 24 hours ago $\leq 0.527$ & 0.4100 & 0.0713 & 5.7517 & $<.001$ \\
\hline Log CO concentration 2 days ago $\leq-0.426$ or $>0.366$ & 0.2789 & 0.0743 & 3.7546 & $<.001$ \\
\hline Log cumulated $\mathrm{CO}$ concentration in the past 5 days $>0.034$ & 1.0558 & 0.2096 & 5.0366 & $<.001$ \\
\hline Log cumulated $\mathrm{CO}$ concentration in the past 7 days $>0.006$ & 0.7773 & 0.2050 & 3.7921 & $<.001$ \\
\hline Log $\mathrm{NO}_{2}$ concentration 6 hours ago & 0.1645 & 0.0763 & 2.1566 & .03 \\
\hline $2.226<\log \mathrm{NO}_{2}$ concentration 18 hours ago $\leq 3.209$ & 0.3400 & 0.0689 & 4.9383 & $<.001$ \\
\hline Log cumulated $\mathrm{NO}_{2}$ cconcentration in the past 1 day $>3.163$ & 0.1880 & 0.0887 & 2.1203 & .03 \\
\hline $\log \mathrm{NO}_{2}$ concentration 2 days ago $<2.695$ & 0.6081 & 0.1352 & 4.4984 & $<.001$ \\
\hline $3.1<\log \mathrm{NO}_{2}$ concentration 3 days ago $\leq 3.6$ & 0.2480 & 0.0727 & 3.4093 & .007 \\
\hline Log $\mathrm{NO}_{2}$ concentration 7 days ago & -0.9739 & 0.1572 & -6.1965 & $<.001$ \\
\hline $\log \mathrm{PM}_{2.5}{ }^{\mathrm{a}}$ concentration 1 day ago $\geq 2.734$ & 0.6872 & 0.1397 & 4.9188 & $<.001$ \\
\hline Log $\mathrm{PM}_{2.5}$ concentration 2 days ago $>3.547$ & 0.2638 & 0.0871 & 3.0294 & .003 \\
\hline $\log \mathrm{PM}_{2.5}$ concentration 3 days ago $\geq 2.598$ & 0.4660 & 0.1869 & 2.4936 & .01 \\
\hline $\log \mathrm{PM}_{2.5}$ concentration 6 days ago $>3.559$ & 0.2502 & 0.0827 & 3.0256 & .003 \\
\hline Log $\mathrm{PM}_{10}{ }^{\mathrm{b}}$ concentration 1 day ago & -0.5895 & 0.1264 & -4.6622 & $<.001$ \\
\hline $0.926<\log \mathrm{SO}_{2}$ concentration $\leq 2.325$ & 0.2053 & 0.0768 & 2.6736 & .008 \\
\hline $0.77<\log \mathrm{SO}_{2}$ concentration 3 hours ago $\leq 1.902$ & 0.1994 & 0.0718 & 2.7766 & .006 \\
\hline $0.73<\log \mathrm{SO}_{2}$ concentration 18 hours ago $\leq 1.819$ & 0.2264 & 0.0683 & 3.3144 & .001 \\
\hline $1.062<\log \mathrm{SO}_{2}$ concentration 24 hours ago $\leq 2.926$ & 0.2025 & 0.0726 & 2.7908 & .005 \\
\hline $0.772<\log \mathrm{SO}_{2}$ concentration 5 days ago $\leq 1.558$ & 0.2316 & 0.0696 & 3.3270 & .001 \\
\hline $0.946<\log \mathrm{SO}_{2}$ concentration 6 days ago $\leq 1.604$ & 0.2500 & 0.0682 & 3.6660 & $<.001$ \\
\hline Log $\mathrm{SO}_{2}$ concentration 7 days ago & 0.5296 & 0.1162 & 4.5578 & $<.001$ \\
\hline
\end{tabular}

${ }^{\mathrm{a}} \mathrm{PM}_{2.5}$ : particulate matter with a diameter of $<2.5 \mu \mathrm{m}$.

${ }^{\mathrm{b}} \mathrm{PM}_{10}$ : particulate matter with a diameter of $<10 \mu \mathrm{m}$. 
Figure 1. Generalized additive model (GAM) plot showing the relationship between systolic blood pressure and CO exposure.

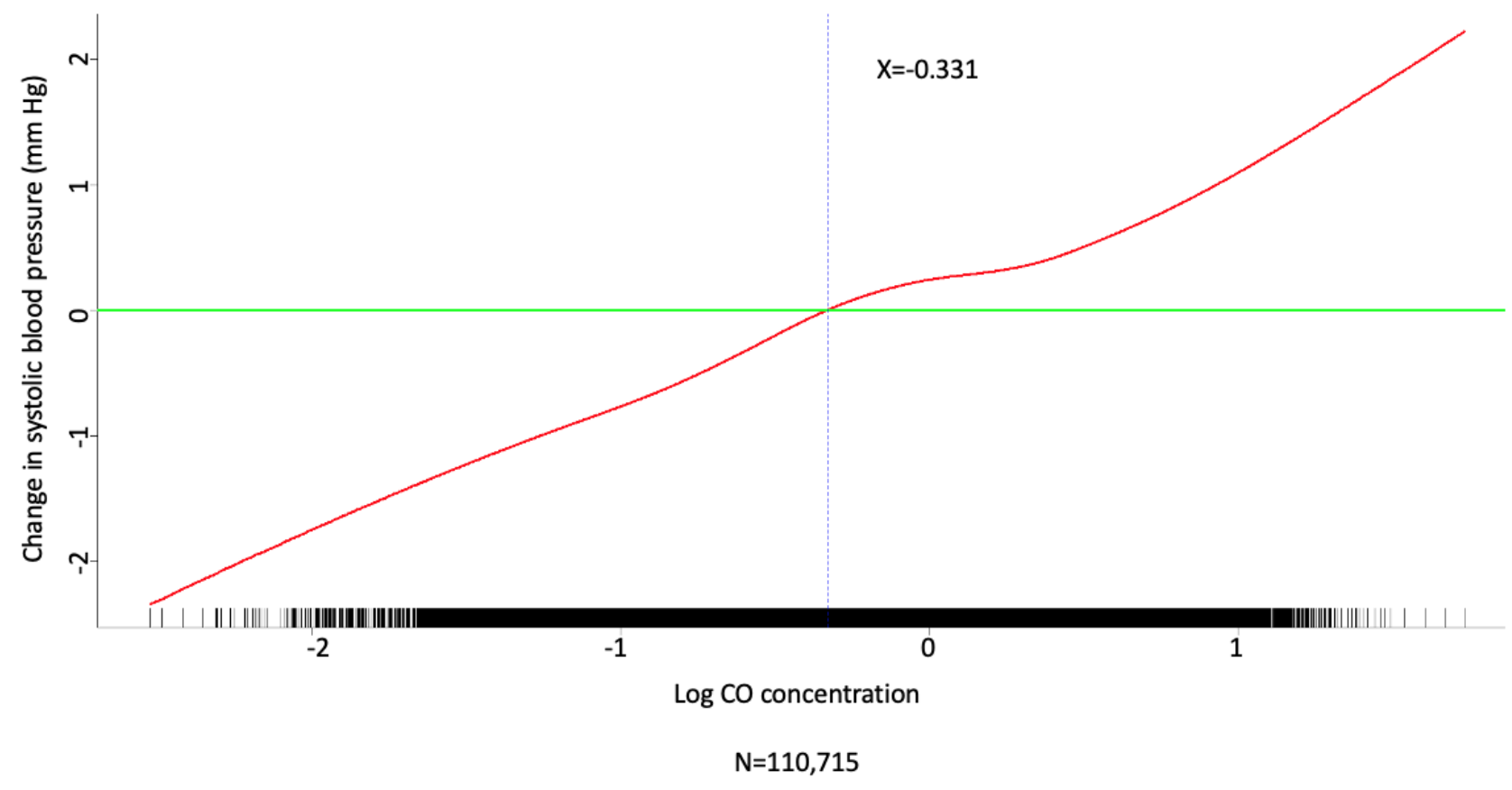

Figure 2. Generalized additive model (GAM) plot showing the relationship between systolic blood pressure and $\mathrm{SO}_{2}$ exposure.

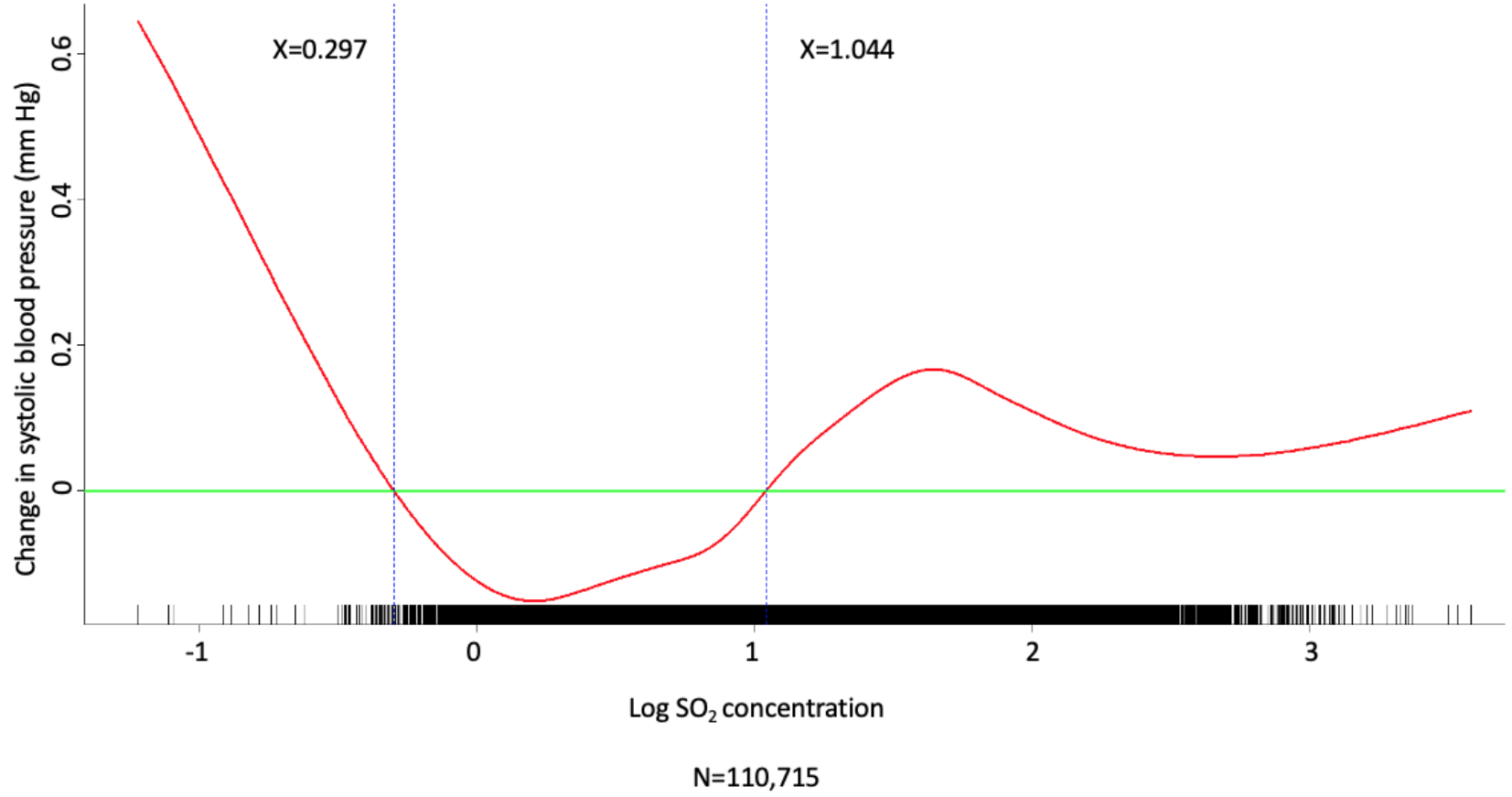


Figure 3. Generalized additive model (GAM) plot showing the relationship between systolic blood pressure and $\mathrm{NO}_{2}$ exposure.

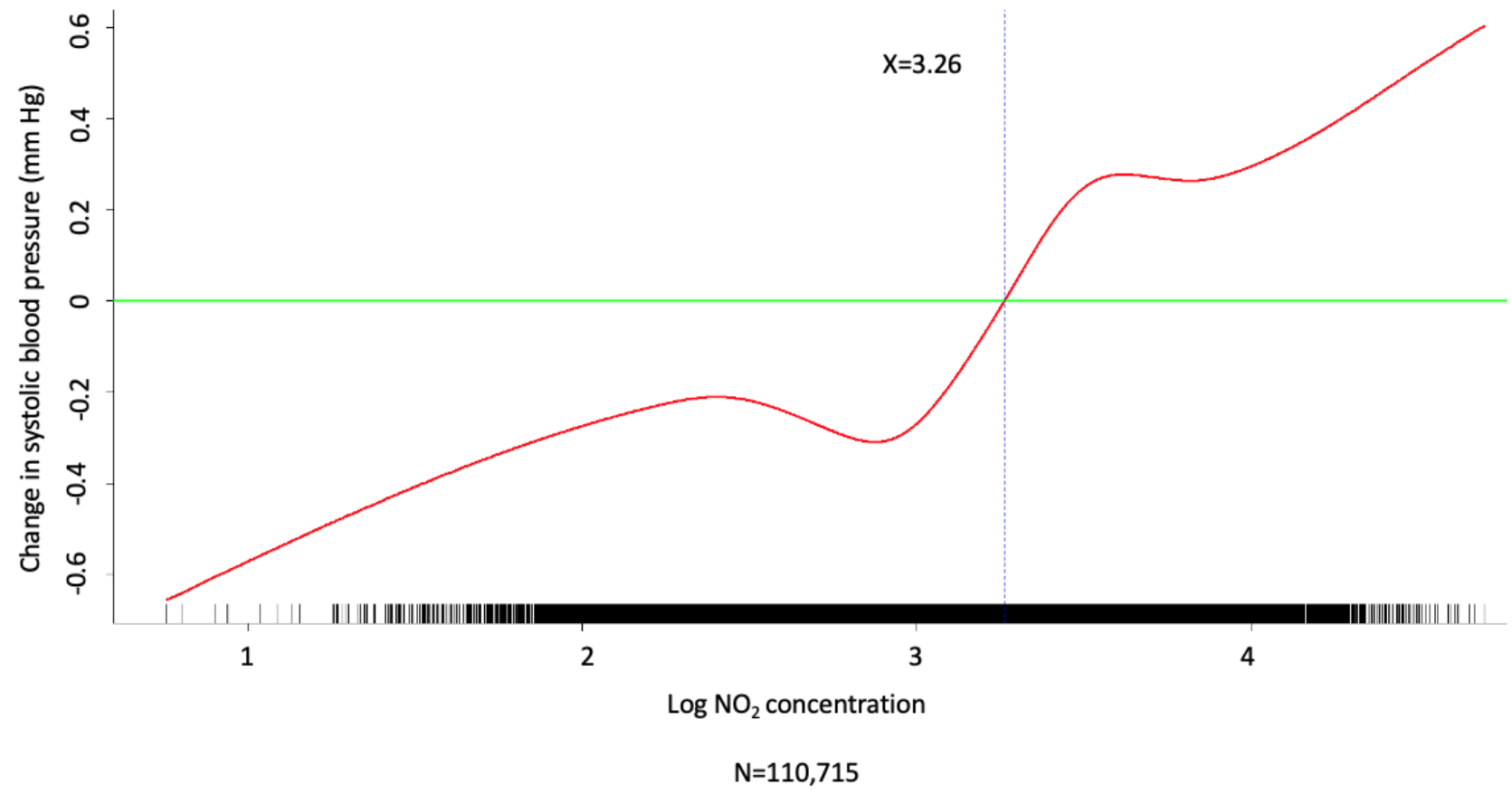

Figure 4. Generalized additive model (GAM) plot showing the relationship between systolic blood pressure and $\mathrm{PM}_{10}$ exposure. $\mathrm{PM}_{10}$ : particulate matter with a diameter of $<10 \mu \mathrm{m}$.

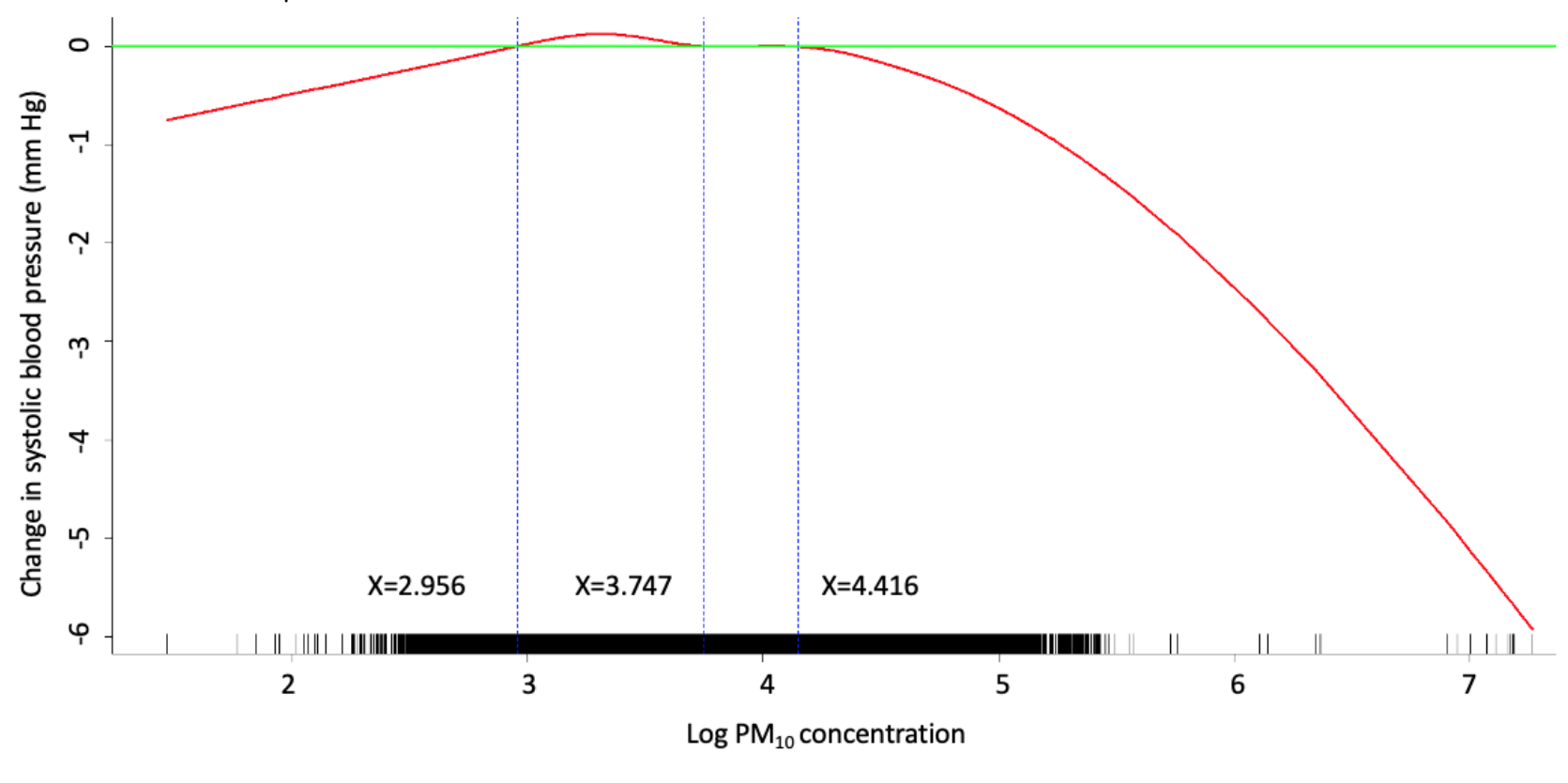

$\mathrm{N}=110,715$ 
Figure 5. Generalized additive model (GAM) plot showing the relationship between systolic blood pressure and $\mathrm{PM}_{2.5}$ exposure. $\mathrm{PM}_{2.5}:$ particulate matter with a diameter of $<2.5 \mu \mathrm{m}$.

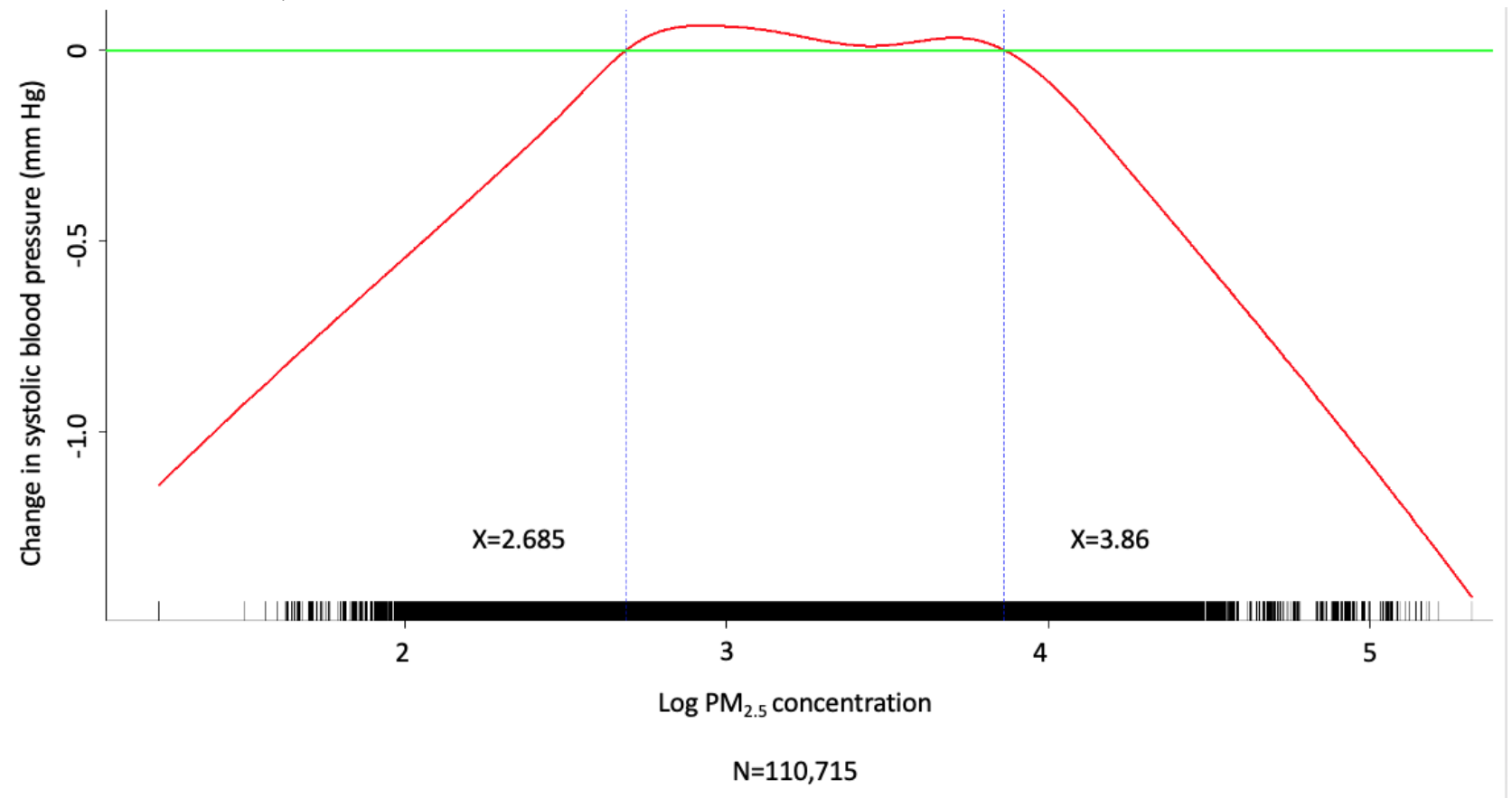

\section{Discussion}

\section{Principal Findings}

Human health is significantly correlated with genomic and environmental factors. Wild [20] defined the "exposome" in 2005 as a concept that "encompasses life-course environmental exposures (including lifestyle factors), from the prenatal period onwards." Among these factors in the exposome, air pollution poses a major threat to human health. Exposure to ambient air pollution, either over the short or long term, increases cardiovascular mortality accordingly $[6,8]$. The detrimental effect of air pollution on BP is considered one of the mechanisms leading to this outcome. However, the association between short-term or long-term exposure to ambient air pollution and BP is inconsistent in the literature. Among the routinely monitored air pollutants, $\mathrm{PM}_{2.5}$ is considered one of the most dangerous agents influencing human health. Brook et al [8] adequately described the association between particulate matter air pollution and CVD, suggesting that exposure to ambient $\mathrm{PM}_{2.5}$ would potentially increase BP. A few mechanisms explaining why $\mathrm{PM}_{2.5}$ increases $\mathrm{BP}$ have been reported before: (1) altering the autonomic nervous system to favor a sympathetic tone [21], (2) increasing oxidative stress through endogenous proinflammatory mediators or vasculo-active molecules [22,23], (3) directly influencing the vascular endothelium [24,25], and (4) inducing abnormal DNA methylation [26,27]. Many studies have investigated the association between exposure to ambient $\mathrm{PM}_{2.5}$ and $\mathrm{BP}$, which has been reported before, but the effect was inconsistent. To date, 4 meta-analyses have addressed this issue. Liang et al [9] reported positive associations among $\mathrm{PM}_{2.5}, \mathrm{SBP}$, and DBP. Zhang et al [10] reported that a high SBP is significantly associated with the exposure to $\mathrm{PM}_{2.5}$, while a high DBP is only associated with the exposure to $\mathrm{PM}_{10}$, but not $\mathrm{PM}_{2.5}$. Cai et al
[11] reported that short-term exposure to $\mathrm{SO}_{2}, \mathrm{PM}_{2.5}$, and $\mathrm{PM}_{10}$ was significantly associated with an increased risk of hypertension. The association between BP and air pollutants other than PM has not been investigated in detail. In a recent meta-analysis by Yang et al [12], short-term exposure to ambient air pollution was significantly associated with hypertension $\left(\mathrm{PM}_{10}, \mathrm{PM}_{2.5}, \mathrm{SO}_{2}\right.$, and $\left.\mathrm{NO}_{2}\right), \mathrm{SBP}\left(\mathrm{PM}_{2.5}\right.$ and $\left.\mathrm{SO}_{2}\right)$, and DBP $\left(\mathrm{PM}_{10}, \mathrm{PM}_{2.5}, \mathrm{SO}_{2}\right.$, and $\left.\mathrm{NO}_{2}\right)$. However, these meta-analyses were mainly performed on the basis of epidemiological studies. To our knowledge, our study is the first to investigate the relationships among demographic, clinical, meteorological factors, air pollutants, and HBP at the patient level.

There are some notable findings in this study. First, at the patient level, ambient air pollution was significantly associated with HBP, both SBP and DBP, and the culprit pollutants included all of the 5 pollutants $\left(\mathrm{PM}_{10}, \mathrm{PM}_{2.5}, \mathrm{SO}_{2}, \mathrm{NO}_{2}\right.$, and $\left.\mathrm{CO}\right)$ that were included in this study. The association between HBP and ambient air pollution was surprisingly nonlinear, given that most previous studies used a linear model to evaluate the effect of ambient air pollution on BP and hypertension. Yang et al [12] suspected that the relationship between ambient air pollution and BP was nonlinear, and this may be the reason why even meta-analyses that have been well performed could not adequately explain the association between ambient air pollution and BP because most previous studies mistakenly used a linear model. One of the strengths of this study was that we considered HBP rather than office BP measurements, which was the case in most of the previous studies. HBP is now considered a more important predictor than office BP for future coronary and cerebral events [28]. All ambient air pollutants investigated in this study significantly affected HBP, while in prior meta-analyses, only some of the ambient air pollutants were significantly associated with hypertension, SBP, and DBP. This could explain why ambient air pollution poses a great hazard 
to human health. Data regarding medication in this study are also important. No study thus far has investigated whether antihypertensive agents play a role in the association between ambient air pollution and BP. Our prior study [18] showed that short-term exposure to low ambient temperature significantly increases HBP, and that this effect could be modified by antihypertensive agents. In this study, the potential confounding effect of medications was also considered, but ambient air pollutants still showed significant associations with HBP. Limited by the statistical model, the exact effect of each class of antihypertensive agents on the relationship between ambient air pollution and HBP could not be fully elucidated. Of note, although there were significant associations between environmental factors and HBP, demographic and clinical factors seemed to play an even more important role in HBP, as shown in Multimedia Appendix 2. Nonetheless, since hypertension has such a marked impact on human health, a holistic approach for BP control, including adequate modification of environmental exposure (both meteorological factors and ambient air pollutants), should be adopted. The importance of air pollution control cannot be overemphasized.

\section{Limitations}

This study has a few limitations. First, this was a retrospective registry with a relatively small number of patients. Second, the study population comprised patients with chronic CVD with great adherence and health insights as they opted to participate in a telehealth care program. Thus, whether the study results can be extrapolated to other patient populations should be carefully considered. Third, individual ambient air pollutant concentrations were calculated using a spatial model that might not reflect true personal exposure. Jiang et al [29] reported that even people who were geographically close could have distinct personalized exposomes. Without precise assessment of personal environmental exposure, further evaluation of the associations between the environment and human health seems futile. Jiang et al [29] reported that novel wearable devices that are capable of assessing both biotic and abiotic exposure may be used to solve this problem. As shown in our previous study [18] and in this study, meteorological factors (including temperature, relative humidity, and wind speed) and ambient air pollutants $\left(\mathrm{NO}_{2}, \mathrm{PM}_{10}, \mathrm{PM}_{2.5}, \mathrm{CO}\right.$, and $\left.\mathrm{SO}_{2}\right)$ had significant effects on human health at the patient level and should be considered in future studies on abiotic exposure among exposomes. Fourth, the possible interactions among air pollutants, including synergistic effects and collinearity, were not addressed in this study. Fifth, this study reports a nonlinear relationship between $\mathrm{BP}$ and ambient air pollutants, but further evaluation of the dose-effect curve was limited by the current statistical methods. Similarly, the interactions between ambient air pollution and other environmental factors, such as meteorological factors, were not addressed in this study. Multiscale entropy is a novel nonlinear method that has been applied to predict outcomes in a few clinical settings, including trauma [30], after cardiac arrest on mechanical circulatory support [31], acute stroke [32], and autonomic imbalance after myocardial infarction [33]. Further studies may also apply this method to evaluate the effect of ambient air pollution on BP. Sixth, this study only investigated the effect of short-term exposure to ambient air pollution; the effect of long-term exposure remains unknown. Seventh, ozone is an important air pollutant associated with an elevated BP [34-38]. However, during the study period (2009-2013), ozone was not routinely monitored by the Environmental Protection Administration in Taiwan; hence, it was not feasible to evaluate the relationship between ozone and HBP in the current study. Finally, the lag effect of each air pollutant on BP was not precisely determined in this study because of the complexity of the statistical analysis.

\section{Conclusions}

Short-term exposure to ambient air pollution significantly affects both home SBP and DBP in patients with chronic CVD, and the relationship between ambient air pollution and HBP is nonlinear.

\section{Acknowledgments}

We thank Dr Fu-Chang Hu (Institute of Clinical Medicine, National Taiwan University) for statistical support. This study was funded by the National Taiwan University Hospital (106-A136).

\section{Conflicts of Interest}

None declared.

\section{Multimedia Appendix 1}

More Statistical Details.

[DOCX File, 17 KB-Multimedia Appendix 1]

\section{Multimedia Appendix 2}

Additional figure and figure legends. [DOCX File, 395 KB-Multimedia Appendix 2]

\section{References}


1. Dockery DW, Pope CA, Xu X, Spengler JD, Ware JH, Fay ME, et al. An association between air pollution and mortality in six U.S. cities. N Engl J Med 1993 Dec 09;329(24):1753-1759. [doi: 10.1056/NEJM199312093292401] [Medline: $\underline{8179653}$ ]

2. Balbus JM, Maynard AD, Colvin VL, Castranova V, Daston GP, Denison RA, et al. Meeting report: hazard assessment for nanoparticles--report from an interdisciplinary workshop. Environ Health Perspect 2007 Nov;115(11):1654-1659 [FREE Full text] [doi: 10.1289/ehp.10327] [Medline: 18007999]

3. Health effects of outdoor air pollution. Committee of the Environmental and Occupational Health Assembly of the American Thoracic Society. Am J Respir Crit Care Med 1996 Jan;153(1):3-50. [doi: 10.1164/ajrccm.153.1.8542133] [Medline: 8542133]

4. Health effects of outdoor air pollution. Part 2. Committee of the Environmental and Occupational Health Assembly of the American Thoracic Society. Am J Respir Crit Care Med 1996 Feb;153(2):477-498. [doi: 10.1164/ajrccm.153.2.8564086] [Medline: $\underline{8564086]}$

5. Kaiser J. Epidemiology. Mounting evidence indicts fine-particle pollution. Science 2005 Mar 25;307(5717):1858-1861. [doi: $10.1126 /$ science.307.5717.1858a] [Medline: 15790822 ]

6. Pope CA, Bates DV, Raizenne ME. Health effects of particulate air pollution: time for reassessment? Environ Health Perspect 1995 May;103(5):472-480 [FREE Full text] [doi: 10.1289/ehp.95103472] [Medline: 7656877]

7. Pope CA. Epidemiology of fine particulate air pollution and human health: biologic mechanisms and who's at risk? Environ Health Perspect 2000 Aug;108 Suppl 4:713-723 [FREE Full text] [doi: 10.1289/ehp.108-1637679] [Medline: 10931790]

8. Brook RD, Rajagopalan S, Pope CA, Brook JR, Bhatnagar A, Diez-Roux AV, American Heart Association Council on EpidemiologyPrevention, Council on the Kidney in Cardiovascular Disease, Council on Nutrition, Physical ActivityMetabolism. Particulate matter air pollution and cardiovascular disease: An update to the scientific statement from the American Heart Association. Circulation 2010 Jun 01;121(21):2331-2378. [doi: 10.1161/CIR.0b013e3181dbece1] [Medline: 20458016]

9. Liang R, Zhang B, Zhao X, Ruan Y, Lian HZ, Fan Z. Effect of exposure to PM2.5 on blood pressure: a systematic review and meta-analysis. J Hypertens 2014 Nov;32(11):2130-2141. [doi: 10.1097/HJH.0000000000000342] [Medline: 25250520]

10. Zhang H, Qian J, Zhao H, Wang J, Zhu H, Zhou Y, et al. A study of the association between atmospheric particulate matter and blood pressure in the population. Blood Press 2016 Jun;25(3):169-176. [doi: 10.3109/08037051.2015.1111019] [Medline: 26634767]

11. Cai Y, Zhang B, Ke W, Feng B, Lin H, Xiao J, et al. Associations of Short-Term and Long-Term Exposure to Ambient Air Pollutants With Hypertension: A Systematic Review and Meta-Analysis. Hypertension 2016 Jul;68(1):62-70. [doi: 10.1161/HYPERTENSIONAHA.116.07218] [Medline: 27245182]

12. Yang B, Qian Z, Howard SW, Vaughn MG, Fan S, Liu K, et al. Global association between ambient air pollution and blood pressure: A systematic review and meta-analysis. Environ Pollut 2018 Apr;235:576-588. [doi: 10.1016/j.envpol.2018.01.001] [Medline: 29331891]

13. Anker SD, Koehler F, Abraham WT. Telemedicine and remote management of patients with heart failure. Lancet 2011 Aug 20;378(9792):731-739. [doi: 10.1016/S0140-6736(11)61229-4] [Medline: 21856487]

14. Chen Y, Lin Y, Hung C, Huang C, Yeih D, Chuang P, et al. Clinical outcome and cost-effectiveness of a synchronous telehealth service for seniors and nonseniors with cardiovascular diseases: quasi-experimental study. J Med Internet Res 2013 Apr 24;15(4):e87 [FREE Full text] [doi: 10.2196/jmir.2091] [Medline: 23615318]

15. Ho Y, Yu J, Lin Y, Chen Y, Huang C, Hsu T, et al. Assessment of the cost-effectiveness and clinical outcomes of a fourth-generation synchronous telehealth program for the management of chronic cardiovascular disease. J Med Internet Res 2014 Jun 10;16(6):e145 [FREE Full text] [doi: 10.2196/jmir.3346] [Medline: 24915187]

16. Hung C, Yu J, Lin Y, Chen Y, Huang C, Lee J, et al. Mortality Benefit of a Fourth-Generation Synchronous Telehealth Program for the Management of Chronic Cardiovascular Disease: A Longitudinal Study. J Med Internet Res 2016 May 13;18(5):e102 [FREE Full text] [doi: 10.2196/jmir.5718] [Medline: 27177497]

17. Hung C, Lee J, Chen Y, Huang C, Wu V, Wu H, et al. Effect of Contract Compliance Rate to a Fourth-Generation Telehealth Program on the Risk of Hospitalization in Patients With Chronic Kidney Disease: Retrospective Cohort Study. J Med Internet Res 2018 Jan 24;20(1):e23 [FREE Full text] [doi: 10.2196/jmir.8914] [Medline: 29367185]

18. Huang C, Chen Y, Hung C, Lee J, Hsu T, Wu H, et al. Assessment of the Relationship Between Ambient Temperature and Home Blood Pressure in Patients From a Web-Based Synchronous Telehealth Care Program: Retrospective Study. J Med Internet Res 2019 Mar 04;21(3):e12369 [FREE Full text] [doi: 10.2196/12369] [Medline: 30829574]

19. Air quality guidelines. Global update 2005. Particulate matter, ozone, nitrogen dioxide and sulfur dioxide. World Health Organization: Europe. 2006. URL: https://www.euro.who.int/ data/assets/pdf file/0005/78638/E90038.pdf [accessed 2021-05-28]

20. Wild CP. Complementing the genome with an "exposome": the outstanding challenge of environmental exposure measurement in molecular epidemiology. Cancer Epidemiol Biomarkers Prev 2005 Aug;14(8):1847-1850 [FREE Full text] [doi: 10.1158/1055-9965.EPI-05-0456] [Medline: 16103423]

21. Perez CM, Hazari MS, Farraj AK. Role of autonomic reflex arcs in cardiovascular responses to air pollution exposure. Cardiovasc Toxicol 2015 Jan;15(1):69-78 [FREE Full text] [doi: 10.1007/s12012-014-9272-0] [Medline: 25123706] 
22. Miller M. The role of oxidative stress in the cardiovascular actions of particulate air pollution. Biochem Soc Trans 2014 Aug;42(4):1006-1011. [doi: 10.1042/BST20140090] [Medline: 25109994]

23. Seaton A, MacNee W, Donaldson K, Godden D. Particulate air pollution and acute health effects. Lancet 1995 Jan 21;345(8943):176-178. [doi: 10.1016/s0140-6736(95)90173-6] [Medline: 7741860]

24. Furuyama A, Kanno S, Kobayashi T, Hirano S. Extrapulmonary translocation of intratracheally instilled fine and ultrafine particles via direct and alveolar macrophage-associated routes. Arch Toxicol 2009 May;83(5):429-437. [doi: 10.1007/s00204-008-0371-1] [Medline: 18953527]

25. Geiser M, Kreyling WG. Deposition and biokinetics of inhaled nanoparticles. Part Fibre Toxicol 2010 Jan 20;7:2 [FREE Full text] [doi: 10.1186/1743-8977-7-2] [Medline: 20205860]

26. Bellavia A, Urch B, Speck M, Brook RD, Scott JA, Albetti B, et al. DNA hypomethylation, ambient particulate matter, and increased blood pressure: findings from controlled human exposure experiments. J Am Heart Assoc 2013 Jun 19;2(3):e000212 [FREE Full text] [doi: 10.1161/JAHA.113.000212] [Medline: 23782920]

27. Perera FP, Wang S, Vishnevetsky J, Zhang B, Cole KJ, Tang D, et al. Polycyclic aromatic hydrocarbons-aromatic DNA adducts in cord blood and behavior scores in New York city children. Environ Health Perspect 2011 Aug;119(8):1176-1181 [FREE Full text] [doi: 10.1289/ehp.1002705] [Medline: 21486719]

28. Kario K, Saito I, Kushiro T, Teramukai S, Tomono Y, Okuda Y, et al. Morning Home Blood Pressure Is a Strong Predictor of Coronary Artery Disease: The HONEST Study. J Am Coll Cardiol 2016 Apr 05;67(13):1519-1527 [FREE Full text] [doi: 10.1016/j.jacc.2016.01.037] [Medline: 27150682]

29. Jiang C, Wang X, Li X, Inlora J, Wang T, Liu Q, et al. Dynamic Human Environmental Exposome Revealed by Longitudinal Personal Monitoring. Cell 2018 Sep 20;175(1):277-291.e31 [FREE Full text] [doi: 10.1016/j.cell.2018.08.060] [Medline: $\underline{30241608]}$

30. Norris P, Anderson S, Jenkins J, Williams AJ, Morris JA. Heart rate multiscale entropy at three hours predicts hospital mortality in 3,154 trauma patients. Shock 2008 Jul;30(1):17-22. [doi: 10.1097/SHK.0b013e318164e4d0] [Medline: 18323736]

31. Lin Y, Huang H, Chang Y, Lin C, Lo M, Liu LD, et al. Multi-scale symbolic entropy analysis provides prognostic prediction in patients receiving extracorporeal life support. Crit Care 2014 Oct 24;18(5):548 [FREE Full text] [doi: 10.1186/s13054-014-0548-3] [Medline: 25341381]

32. Tang S, Jen H, Lin Y, Hung C, Jou W, Huang P, et al. Complexity of heart rate variability predicts outcome in intensive care unit admitted patients with acute stroke . J Neurol Neurosurg Psychiatry 2015 Jan;86(1):95-100. [doi: 10.1136/jnnp-2014-308389] [Medline: 25053768]

33. Hung C, Huang C, Pan S, Ma H, Huang C, Guo YL, et al. Acute particulate matter exposure is associated with disturbances in heart rate complexity in patients with prior myocardial infarction. Sci Total Environ 2020 Sep 01;733:138842. [doi: 10.1016/j.scitotenv.2020.138842] [Medline: 32446047]

34. Chen Q, Li H, Liu Q, Wang W, Deng F, Sun Z, et al. Does psychosocial stress modify the association of fine particulate matter and ozone with cardiovascular health indicators? Environ Pollut 2021 May 15;277:116726. [doi:

10.1016/j.envpol.2021.116726] [Medline: 33639598]

35. Kaufman JD, Elkind MS, Bhatnagar A, Koehler K, Balmes JR, Sidney S, American Heart Association Advocacy Coordinating Committee. Guidance to Reduce the Cardiovascular Burden of Ambient Air Pollutants: A Policy Statement From the American Heart Association. Circulation 2020 Dec 08;142(23):e432-e447. [doi: 10.1161/CIR.0000000000000930] [Medline: $\underline{33147996]}$

36. Khajavi A, Tamehri Zadeh SS, Azizi F, Brook RD, Abdi H, Zayeri F, et al. Impact of short- and long-term exposure to air pollution on blood pressure: A two-decade population-based study in Tehran. Int J Hyg Environ Health 2021 May;234:113719. [doi: 10.1016/j.ijheh.2021.113719] [Medline: 33677362]

37. Song J, Zhu J, Tian G, Li H, Li H, An Z, et al. Short time exposure to ambient ozone and associated cardiovascular effects: A panel study of healthy young adults. Environ Int 2020 Apr;137:105579 [FREE Full text] [doi: 10.1016/j.envint.2020.105579] [Medline: $\underline{\text { 32086080] }}$

38. Song L, Smith GS, Adar SD, Post WS, Guallar E, Navas-Acien A, et al. Ambient air pollution as a mediator in the pathway linking race/ethnicity to blood pressure elevation: The multi-ethnic study of atherosclerosis (MESA). Environ Res 2020 Jan;180:108776. [doi: 10.1016/j.envres.2019.108776] [Medline: 31639655]
Abbreviations
BP: blood pressure
CVD: cardiovascular disease
DBP: diastolic blood pressure
GAM: generalized additive models
HBP: home blood pressure
NTUH: National Taiwan University Hospital
$\mathbf{P M}_{10}$ : particulate matter with a diameter of $<10 \mu \mathrm{m}$
$\mathbf{P M}_{2.5}$ : particulate matter with a diameter of $<2.5 \mu \mathrm{m}$ 
SBP: systolic blood pressure

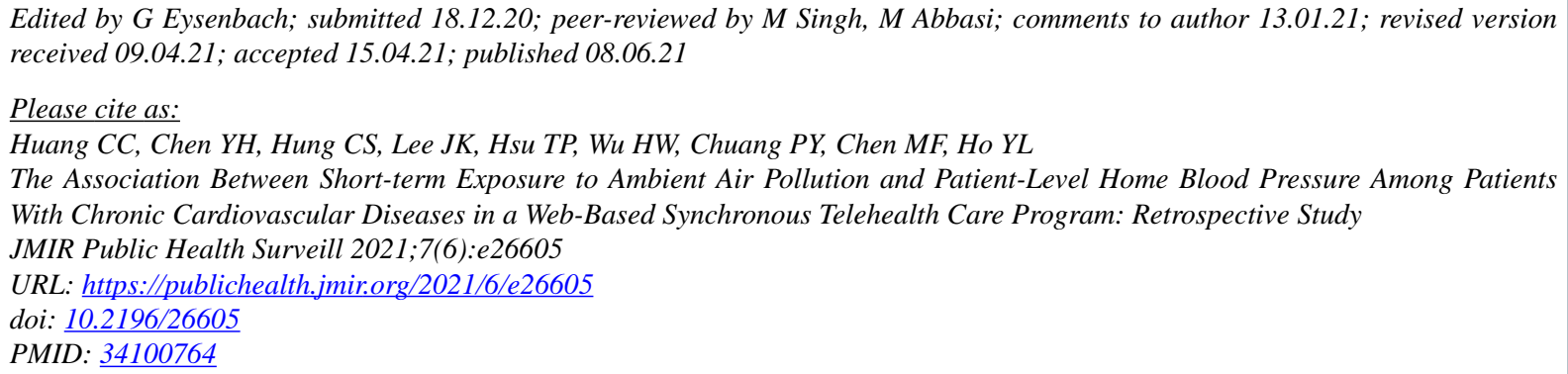

(C) Ching-Chang Huang, Ying-Hsien Chen, Chi-Sheng Hung, Jen-Kuang Lee, Tse-Pin Hsu, Hui-Wen Wu, Pao-Yu Chuang, Ming-Fong Chen, Yi-Lwun Ho. Originally published in JMIR Public Health and Surveillance (https://publichealth.jmir.org), 08.06.2021. This is an open-access article distributed under the terms of the Creative Commons Attribution License (https://creativecommons.org/licenses/by/4.0/), which permits unrestricted use, distribution, and reproduction in any medium, provided the original work, first published in JMIR Public Health and Surveillance, is properly cited. The complete bibliographic information, a link to the original publication on https://publichealth.jmir.org, as well as this copyright and license information must be included. 